\title{
Spatio-Temporal Analysis of EEG Signal during Consciousness using Convolutional Neural Network
}

\author{
Minji Lee ${ }^{1, *}$, Seul-Ki Yeom ${ }^{1, *}$, Benjamin Baird ${ }^{2}$, Olivia Gosseries ${ }^{2,3,4}$, \\ Jaakko O. Nieminen ${ }^{2,5}$, Giulio Tononi ${ }^{2}$, and Seong-Whan Lee ${ }^{1}$ \\ ${ }^{1}$ Department of Brain and Cognitive Engineering, Korea University, Seoul, Korea \\ ${ }^{2}$ Department of Psychiatry, University of Wisconsin, Madison, USA \\ ${ }^{3}$ Department of Psychology, University of Wisconsin, Madison, USA \\ ${ }^{4}$ Coma Science Group, GIGA-Research \& Neurology Department, University and University Hospital of Liege, Liege, Belgium \\ ${ }^{5}$ Department of Neuroscience and Biomedical Engineering, Aalto University School of Science, Espoo, Finland \\ minjilee@korea.ac.kr, skyeom@korea.ac.kr, bbaird@wisc.edu, ogosseries@ulg.ac.be, jaakko.nieminen@aalto.fi, \\ gtononi@wisc.edu, sw.lee@korea.ac.kr
}

\begin{abstract}
Electroencephalogram (EEG) measurement could help to distinguish the level of consciousness in an individual without requiring a behavioral response, which could be useful as a diagnostic aid in patients with disorders of consciousness. In this study, we explored the EEG-evoked perturbation and analyzed consciousness using event-related spectral perturbation and convolutional neural network. We observed a novel EEG neurophysiological signature that can be used to monitor brain activity during unconsciousness. Also, the performance accuracy in the parietal region was higher than in the frontal region. The sensitivity for conscious experience was $90.9 \%$, whereas sensitivity for unconscious experience was at the chance level in the parietal region. These results could be evidence for the importance of the posterior hot zone and could help shed light on the internal neural dynamics related to conscious experience.
\end{abstract}

Keywords-sedation; sleep; consciousness; classification; transcranial magnetic stimulation

\section{INTRODUCTION}

Sleep classification has been traditionally performed by experts using electroencephalography (EEG) signals. The automatic classification of sleep stage has been studied based on machine learning [1]. In addition to automatically classifying the stage of sleep, it is also of interest to be able to classify whether an individual is having conscious experiences during sleep (i.e., in the form of dreams). Classification of consciousness using passive measures of brain activity has applications, and could be useful as diagnostic aids for patients who are non-communicative due to traumatic injury or neurodegenerative disease. Traditionally, dreaming was considered to occur during rapid eye-movement (REM) sleep, but research has shown that dreaming also occurs during nonrapid eye-movement (NREM) sleep [2]. Both REM and NREM sleep therefore present an opportunity to study the changes in neural dynamics associated with consciousness.

Conscious experience within the same sleep stage was recently investigated using a serial awakening paradigm [3]. Conscious experience was related to local decreases of brain activity in low-frequency oscillations over a so-called "posterior hot zone" (which included regions of occipital and

This work was supported by Institute for Information \& Communication Technology Promotion (IITP) grant funded by the Korea government (No. 2017-0-00451, Development of BCI based Brain and Cognitive Computing Technology for Recognizing User's Intentions using Deep Learning). parietal cortex), rather than anterior brain regions. Highfrequency activity in these regions also correlated with specific dream contents. These data suggest that activity in the posterior hot zone may underlie the crucial difference between consciousness and unconsciousness [4]. Based on these data, the authors also predicted the presence of consciousness and unconsciousness during NREM sleep. The prediction accuracy was $86.2 \%$ for dream experiences during NREM sleep [2]. However, this predictive method only targeted the most extreme EEG states over the course of the night, specifically only when a bi-spectral threshold was reached indicating that high-frequency activity was in the highest $15 \%$ and simultaneously low-frequency activity was in the lowest $15 \%$ for a given participant throughout the night.

Combining transcranial magnetic stimulation (TMS) and high density EEG (TMS-hdEEG) has made it possible to measure cortical changes in thalamo-cortical circuits over the past 10 years [5]. This method holds promise for distinguishing the levels of consciousness in patients with disorders of consciousness that was not possible with simple EEG analysis [6]. TMS-evoked EEG responses have been shown to differ depending on whether an individual reports having a conscious experience during NREM sleep. Specifically, TMS causes a larger negative deflection in the no conscious experience [7]. However, no studies have attempted to predict conscious experience during sleep using TMS perturbation.

Recent studies have used deep learning in the fields of brain-computer interfaces. The convolutional neural network $(\mathrm{CNN})$, a kind of deep learning, is suitable for classifying EEG signals because EEG has characteristics which are many variations over time and individuals [8]. However, CNN has not been used for classification of conscious experience.

In this study, we used the $\mathrm{CNN}$ architecture for classifying conscious experience within NREM sleep for the first time. Specifically, we analyzed perturbational changes after TMS in both anterior and posterior scalp regions and used $\mathrm{CNN}$ to attempt to classify the presence and absence of conscious experience.

* These authors contributed equally to this work 
TABLE I. INFORMATION OF THE EXPERIMENTAL DATA

\begin{tabular}{|c|c|c|c|}
\hline Condition & CE & NCE & Total \\
\hline $\begin{array}{c}\text { Num. of } \\
\text { awakenings }\end{array}$ & 108 & 78 & 186 \\
\hline
\end{tabular}

\section{METHODS}

\section{A. Data Description and Preprocessing}

The experimental data used in this study have been described elsewhere [7]. In brief, six right-handed healthy subjects participated in this study ( 5 males, age $23.7 \pm 3.2$ years). When participants had been in stable NREM sleep for longer than 3 minutes, TMS was applied over a posterior scalp region (above precuneus). Following the TMS pulse sequence, participants were awakened by auditory stimuli and completed a structured questionnaire regarding whether they had been having a conscious experience (i.e., experiencing a dream) before awakening. Responses were divided two conditions: conscious experience (CE) (regardless of recall of contents), and no conscious experience (NCE). Participants were recorded for four or five overnight sessions in the sleep laboratory. The total number of awakenings was 108 and 78 for CE and NCE respectively (Table I).

Data analysis was performed using MATLAB 2017a and EEGLAB toolbox [9]. EEG data were scored according to the AASM manual to classify sleep stage [10]. TMS-related artifacts were removed by linearly interpolating the first $15 \mathrm{~ms}$ of the data post-TMS. EEG data were bandpass filtered between 1.5 and $50 \mathrm{~Hz}$ and down-sampled to $362.5 \mathrm{~Hz}$. Other artifacts, including movement related artifacts, were rejected manually. Finally, data were baseline-corrected using a -400 to 0 msec window and average-referenced.

\section{B. Feature Extraction}

The event-related spectral perturbation (ERSP) was calculated from the preprocessed EEG data. The number of cycles in each wavelet was 3 and wavelet cycles increased with frequency beginning at wave cycles with 0.5 . We also applied bootstrap for significance test. The significance level set to 0.01 and the number of bootstrap replications to accumulate was 1000. In particular, we focused on $\mathrm{Fz}$ and $\mathrm{Pz}$ electrodes for exploring the roles in the anterior and posterior regions.

\section{Convolutional Neural Network Architecture}

The input image was $120 \times 120$ from the EEG signals in the time-frequency analysis for the network. In the time domain, EEG signals were from -400 to $400 \mathrm{msec}$ based on TMS. In the frequency domain, the range was from 1.5 to $50 \mathrm{~Hz}$.

A CNN with the following architecture was trained on the dataset (Table II and Fig. 1). In the convolutional layer, the filter size was 5-by-5 and the number of filter 30 applied zeropadding. The feature map was the same by adding padding. The activation function was the rectified linear unit (ReLU) commonly used. Finally, the fully connected layer which connects to all the neurons was followed. The output size was 2 corresponding to 2 classes for conscious experience. The softmax activation normalized the outcome of the fully connected layer. This layer was used as the probability of conscious experience. The CNN was first trained using stochastic gradient descent with momentum with a momentum of 0.9 learning rate of 0.00001 . The maximum number of epochs set to 50 .

TABLE II. CNN ARCHITECTURE

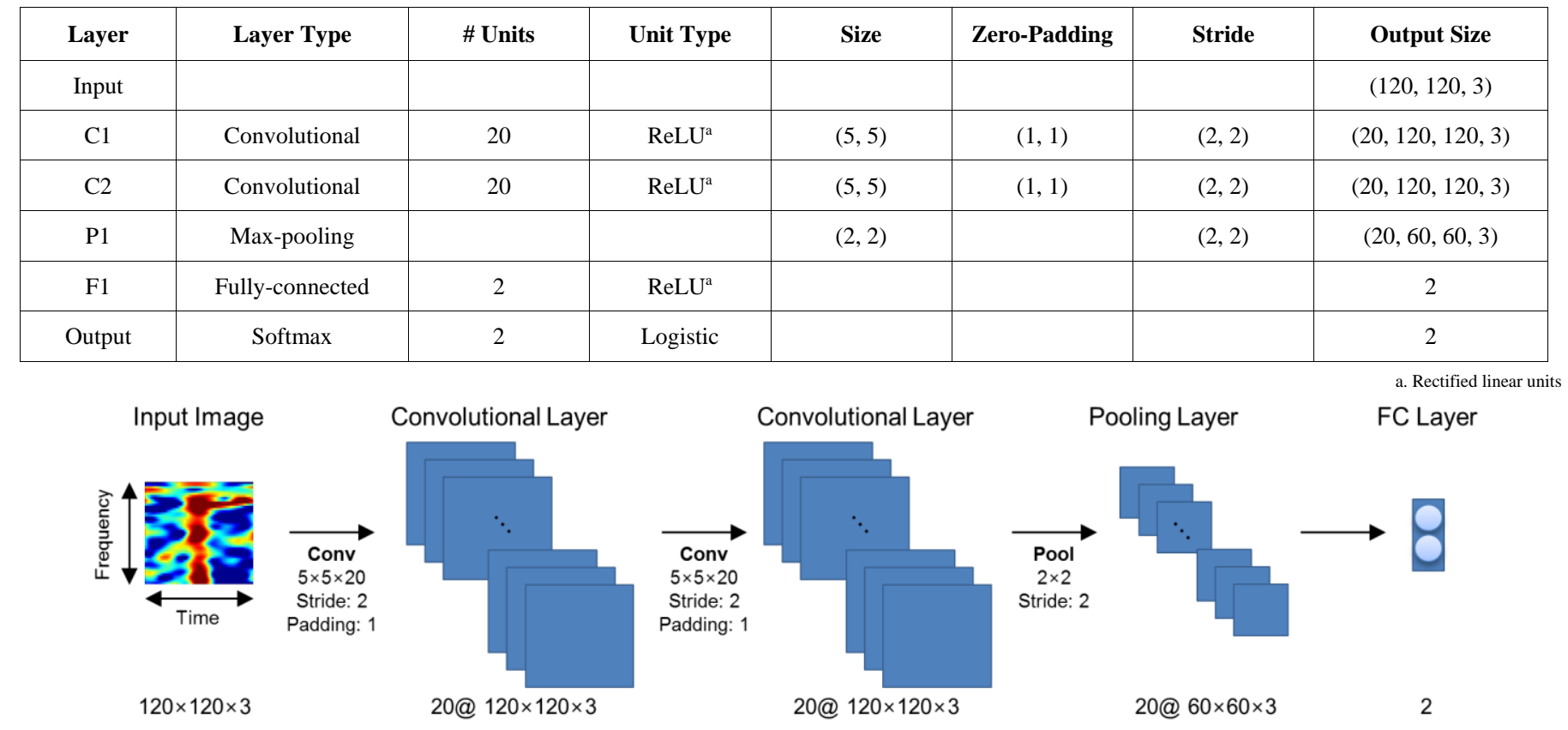

Figure 1. Proposed CNN architecture. 


\section{a. Fz electrode}

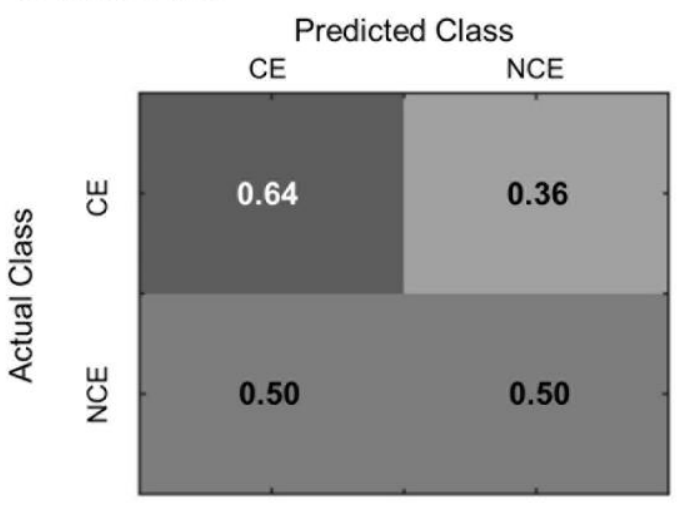

\section{b. Pz electrode}

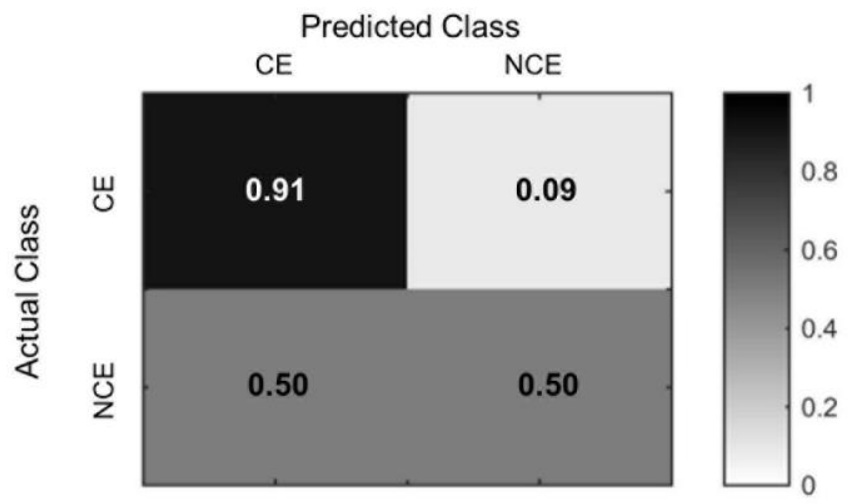

Figure 2. Confusion marices for classification performance.

\section{Evaluation}

We randomly split training and evaluation sets at a 9:1 ratio. In order to compare the prediction performance, the accuracy of each channel and sensitivity according to conscious experience were obtained.

\section{RESULTS}

We investigated the TMS-evoked spectral dynamics and classified the presence and absence of conscious experience based on these dynamics within NREM sleep.

\section{A. Perturbational Dynamics based on TMS}

In both $\mathrm{Fz}$ and $\mathrm{Pz}$ electrodes, the event-related dynamics for TMS at high-frequency were strongly increased ( $\mathrm{p}<0.001)$. The brain activity at $\mathrm{Pz}$ electrode showed stronger negative deflection compared to Fz electrode ( $\mathrm{p}<0.001)$.

\section{B. Classification Performance}

The classification accuracy was $57.8 \%$ at Fz electrode and $73.6 \%$ at $\mathrm{Pz}$ electrode, respectively. The sensitivity was $63.6 \%$ for $\mathrm{CE}$ and $50 \%$ for $\mathrm{NCE}$ over the $\mathrm{Fz}$ electrode, whereas the sensitivity was $90.9 \%$ for $\mathrm{CE}$ and $50 \%$ for NCE over the $\mathrm{Pz}$ electrode (Fig. 2).

\section{DISCUSSION AND CONCLUSION}

In summary, this is the first attempt to classify conscious experience within NREM sleep using CNN. Classification of the EEG signal at a posterior electrode site yielded high classification accuracy for predicting conscious experience. The higher accuracy in the parietal region compared to the frontal region suggests that dynamic changes in posterior cortex may be related to consciousness [2,7]. The sensitivity for conscious experience showed that EEG signals in the conscious states have more predictable characteristics compared to unconscious states [11]. These findings could be helpful for developing new diagnostic tools for assessing the level of consciousness in individuals suffering from disorders of consciousness as a result of stroke, brain injury or neurodegenerative disease.

\section{ACKNOWLEDGMENT}

The authors thank M. Boly for help with the data collection and useful discussions.

\section{REFERENCES}

[1] T. L. T. da Silveira, A. J. Kozakevicius, and C. R. Rodrigues, "Single-channel EEG sleep stage classification based on a streamlined set of statistical features in wavelet domain," Med. Biol. Eng. Comput. Heidelberg, vol. 55, pp. 343-352, Feb. 2017.

[2] F. Siclari et al., "The neural correlates of dreaming," Nat. Neurosci. New York, vol. 20, pp. 872-881, June 2017.

[3] F. Siclari, J. J. LaRocque, B. R. Postle, and G. Tononi, "Assessing sleep consciousness within subjects using a serial awakening paradigm," Front Psychol. Lausanne, vol. 4, pp. 1-9, Aug. 2013.

[4] C. Koch, M. Massimini, M. Boly, and G. Tononi, "Posterior and anterior cortex - where is the difference that makes the difference?," Nat. Rev. Neurosci. London, vol. 17, pp. 105, Oct. 2016.

[5] M. Massimini, F. Ferrarelli, S. Sarasso, and G. Tononi, "Cortical mechanisms of loss of consciousness: insight from TMS/EEG studies," Arch. Ital. Biol. Pisa, vol. 150, pp. 44-55, June 2012.

[6] S. Sarasso et al., "Quantifying cortical EEG responses to TMS in (un)consciousness," Clin. EEG Neurosci. Thousand Oaks, vol. 45, pp. 40-49. Jan. 2014

[7] J. O. Nieminen et al., "Conciousness and cortical responsiveness: a within-state study during non-rapid eye movement sleep," Sie. Rep. London, vol. 6, pp. 1-10, Aug. 2016.

[8] H. Cecotti and A. Graser, "Convolutional neural networks for P300 detection with application to brain-computer interfaces," IEEE Trans. Pattern Anal. Mach. Intell. Los Alamitos, vol. 33, pp. 433-435, Mar. 2011.

[9] A. Delorme, and S. Makeig, "EEGLAB: an open source toolbox for analysis of single-trial EEG dynamics including independent component analysis," J. Neurosci. Methods. Amsterdam, vol. 134, pp. 9-21, Mar. 2004

[10] R. B. Berry et al., "Rules for scoring respiratory events in sleep: update of the 2007 AASM manual for the scoring of sleep and associated events," J. Clin. Sleep Med. Westchester, vol. 8, pp. 597-619, Oct. 2012.

[11] J. Yoo, J. Kwon, and Y. Choe, "Predictable internal brain dynamics in EEG and its relation to conscious states," Front. Neurorobotics. Lausanne, vol. 8, pp. 1-7, June 2014. 